\title{
Comparative Efficacy and Safety of Vancomycin, Linezolid, Tedizolid, and Daptomycin in Treating Patients with Suspected or Proven Complicated Skin and Soft Tissue Infections: An Updated Network Meta-Analysis
}

Jingjuan Feng $\cdot$ Feng Xiang $\cdot$ Jian Cheng $\cdot$ Yeli Gou $\cdot$ Jun Li (D

Received: February 16, 2021 / Accepted: May 7, 2021 / Published online: June 18, 2021

(C) The Author(s) 2021

\begin{abstract}
Introduction: Skin and soft structure infections (SSTIs) caused by methicillin-resistant Staphylococcus aureus (MRSA) pose serious health risks and cause significant cost burdens, and a conclusive recommendation about antibiotics has not yet been generated. Therefore, we performed this updated network meta-analysis to determine the preferred drug for the treatment of MRSA-caused SSTIs.

Methods: We searched PubMed, Embase, and Cochrane Library to identify any potentially eligible randomized controlled trials (RCTs) investigating the comparative efficacy and
\end{abstract}

Jingjuan Feng and Feng Xiang are the co-first authors.

Supplementary Information The online version contains supplementary material available at https:// doi.org/10.1007/s40121-021-00456-0.

J. Feng · J. Li ( $₫)$

School of Clinical Medical Sciences, Southwest Medical University, Luzhou 646000, Sichuan, China e-mail: junli2002@126.com

J. Feng · F. Xiang $\cdot$ J. Cheng $\cdot$ Y. Gou Department of Chinese Medicine Surgery, Sichuan Provincial People's Hospital, University of Electronic Science and Technology of China, Chengdu 610072, Sichuan, China

F. Xiang · J. Cheng · Y. Gou

Chinese Academy of Sciences Sichuan Translational Medicine Research Hospital, Chengdu 610072,

Sichuan, China safety of any two of vancomycin, linezolid, tedizolid, and daptomycin in MRSA-caused SSTIs. All statistical analyses were conducted with RevMan, ADDIS, and STATA software.

Results: Twenty eligible RCTs involving 7804 patients were included for the final analysis. Direct meta-analysis suggested that linezolid was superior to vancomycin in improving clinical (odds ratio [OR], 1.46; 95\% confidence interval $[\mathrm{CI}], 1.07-1.99 ; P=0.02$ ) and microbiological (OR, 1.89; 95\% CI, 1.24-2.86; $P=0.003$ ) success, which were all confirmed by network meta-analyses. No statistical differences were identified regarding other comparisons. Meanwhile, there were no significant differences between any two antibiotics related to safety. Moreover, ranking probabilities indicated that linezolid had the highest probability of being ranked best in terms of clinical and microbiological success.

Conclusion: Based on the limited evidence, linezolid may be a preferred antibiotic for the treatment of MRSA-caused SSTIs because it showed superiority in clinical and microbiological success without difference regarding safety.

Keywords: Skin and soft tissue infection; Methicillin-resistant Staphylococcus aureus; Systematic review; Network meta-analysis 


\section{Key Summary Points}

Twenty eligible RCTs involving 7804 patients were included for the final analysis.

The study suggested that linezolid was superior to vancomycin in improving clinical success.

No statistical differences were identified regarding other comparisons.

There were no significant differences between any two antibiotics about safety.

\section{DIGITAL FEATURES}

This article is published with digital features, including a summary slide, to facilitate understanding of the article. To view digital features for this article go to https://doi.org/10.6084/ m9.figshare.14554872.

\section{INTRODUCTION}

Skin and soft tissue infections (SSTIs) are one of the major threatening questions faced by patients in both the community and hospital settings [1]. It is reported that the methicillinresistant Staphylococcus aureus (MRSA) infections account for $>60 \%$ of SSTIs in most institutions [2]. Meanwhile, the incidence of infections caused by MRSA has been sharply rising recently $[3,4]$. It is critically important that SSTIs caused by MRSA are associated with increased incidence of several complications, which increase the mortality, length of hospital (LOS), and total cost burden [5-7]. Obviously, development of an effective treatment regime for MRSA SSTIs has become an urgent challenge.

Orally or intravenously administered antibiotics still play a critical role in treating MRSA infections, especially complicated SSTIs (cSSTIs) [8]. As the gold standard regime, vancomycin

has been historically used to treat MRSA SSTIs [6]; however, the emergence of vancomycin-resistant $S$. aureus challenges the usage of this regime $[4,9]$. Therefore, several novel antibiotics have been introduced to combat the evolving resistance of this challenging pathogen [8]. Of these antibiotics, linezolid, daptomycin, and tedizolid have been approved to treat MRSA infections [10].

Although some clinical studies have been performed to investigate the role of linezolid, daptomycin, and tedizolid in treating SSTIs [11-13], conflicting results directly limited the clinical decision. Several meta-analyses have also been conducted to systematically determine the optimal regime for SSTIs; however, a conclusive finding has not yet been generated [1, 14-16]. Compared to traditional head-tohead meta-analysis, which only can perform a single comparison at a time, network metaanalysis has been developed and then extensively applied in practice because this method can simultaneously combine multiple evidence including direct and indirect evidence estimated from the available direct comparisons to generate more reliable and robust findings [17]. Although two network meta-analyses $[14,16]$ have also partially considered this topic, we must recognize that some limitations such as an insufficient number of eligible studies have impaired the reliability and robustness of pooled results. Therefore, we performed this updated network meta-analysis to compare these four different antibiotics including vancomycin, linezolid, tedizolid, and daptomycin used for the treatment of MRSA SSTIs to further determine which should be preferentially prescribed.

\section{METHODS}

We designed and then performed the current network meta-analysis according to the framework proposed by the Cochrane Collaboration [18]. However, we did not register the formal protocol for this meta-analysis. Then, we reported all findings in accordance with the Preferred Reporting Items for Systematic Reviews and Meta-analyses (PRISMA) for 
Network Meta-Analysis guidelines [19], summarized in Table S1. In the current network meta-analysis, no ethical approval or patient informed consent was required because this article is based on previously conducted studies and does not contain any new studies with human participants or animals performed by any of the authors.

\section{Identification of Studies}

In the current network meta-analysis, we assigned two independent reviewers to perform a systematic search to identify any potentially eligible studies in PubMed, EMBASE, and the Cochrane Library from their inception to October 2020. We combined the medical subject heading $(\mathrm{MeSH})$ with text to construct the search strategy and then modified it according to the unique requirements of the individual database. We summarized the details of search strings of all targeted databases in Table S2. We only considered the studies published in English and Chinese language for inclusion. Moreover, we also manually checked the references of all included studies and meta-analyses focused on the same topic to capture additional eligible studies. Any divergences on identification of studies were resolved based on the consensus principle or by consulting a third senior reviewer.

\section{Selection Criteria}

According to the previous meta-analysis [16], we designed the following selection criteria: (1) adult patients who were diagnosed with suspected or confirmed MRSA-related infections; (2) patients who were instructed to orally or parenterally use antibiotics with anti-MRSA activity; (3) only randomized controlled trials (RCTs) were considered eligible. Moreover, we only considered the latest study with more sufficient data when a series of studies had been published by the same research group based on the same sample. We excluded studies when: (1) they focused on the preventive effect of antibiotics on colonization or infection; (2) they were designed to investigate the pharmacokinetic or pharmacodynamic of antibiotics; (3) they only investigated the pharmacoeconomics or obtained pooled results of previous studies; (4) they were reviews, editorials, letters, case reports, conference abstracts, and cell and animal studies. Two reviewers independently completed the process of selecting studies. Any divergences about the selection of studies were resolved based on the consensus principle or by consulting a third senior reviewer.

\section{Data Extraction}

Two independent reviewers were assigned to use a standard data extraction sheet to extract essential information as follows: basic information of studies including the lead author's name, publication year, country of the lead author, study design (multiple or single center), basic information of participants including sample size (male/total) and mean age, basic information on treatment regime including details of treatment, treatment duration, and outcomes, and details of risk of bias. Any divergences were solved based on the consensus principle or by consulting a third senior reviewer.

\section{Outcomes of Interest}

In the current network meta-analysis, we considered clinical success, microbiological success, and adverse events (AEs) including drug-related AEs and serious AEs. Clinical success was evaluated to be cured and improved status at test of cure (TOC) in the modified intention-to-treat (mITT) population, which was defined as the randomized patients receiving at least one dose of the study drug. Cured was defined as resolution of the clinical signs and symptoms of infection compared with baseline; improved was defined as improvement in two or more, but not all, clinical signs and symptoms of infection compared with baseline [16].

\section{Quality Assessment}

Two reviewers used the Cochrane Risk of Bias assessment tool [20] to independently assess the 
quality of individual studies using the following six domains: random sequence generation; allocation concealment; blinding of participants, personnel, and outcome assessors; incomplete outcome data; selective reporting; other bias. We labeled a study as low risk of bias if all domains were fulfilled. We labeled a study as high risk of bias if more than one of all domains were not fulfilled. A study was labeled with unclear risk of bias when there was not sufficient information for determination. Any divergences related to quality assessment were resolved based on the consensus principle or by consulting a third senior reviewer.

\section{Statistical Analysis}

In the current study, we simultaneously performed head-to-head meta-analysis and network meta-analysis to compare the comparative effects of four different antibiotics. For head-tohead meta-analysis, we adopted Review Manager (RevMan) 5.3 (Cochrane Collaboration, Copenhagen, Denmark) to complete all statistical analyses based on the random-effects model adopted by Der Simonian-Laired. We calculated the odds ratio (OR) with $95 \%$ confidence interval (CI) to express estimates because all outcomes in the current study were dichotomous data. We first qualitatively inspected the heterogeneity across studies using the Cochrane $Q$ statistic ( $P$ value), and then we used the $I^{2}$ statistic to quantitatively estimate the proportion of heterogeneity except for random error. If $I^{2}<50 \%$ and $P>0.1$, studies were considered to be homogeneous. In contrast, studies were defined as heterogeneous when $I^{2} \geq 50 \%$ and $P<0.1$.

After completing head-to-head meta-analysis, we performed Bayesian network analysis using the Aggregate Data Drug Information System (ADDIS V.1.16.8, Drugis, Groningen, $\mathrm{NL}$ ), which was designed based on the Markov Chain Monte Carlo (MCMC) method [21]. All estimates were expressed as OR with 95\% credible interval (CrI). We performed random effect and consistency models based on the following parameters: (1) 4 chains; (2) 20,000 tuning iterations; (3) 50,000 simulation iterations; (4) thinning interval of 10; (5) 10,000 inference samples; (6) variance scaling factor of 2.5. We used the Brooks-Gelman-Rubin method to evaluate the convergence based on the potential scale reduction factor (PSRF). A PSRF close to 1 indicates that an approximate convergence has been reached, while a PSRF of $<1.2$ is considered acceptable. We did not assess the inconsistencies between the direct and indirect effect because no first loop was constructed in the current study. Meanwhile, we also used STATA software version 14.0 (Stata Corp LP, College Station, TX, USA) to generate an evidence plot. Finally, we used Microsoft Excel to draw ranking probabilities for all the interventions according to the results from ADDIS software.

\section{Publication Bias}

We drew funnel plots regarding clinical and microbiological success to qualitatively inspect the possible presence of publication bias because the accumulated eligible numbers of analyzed studies for these two outcomes were all $>10$ [22].

\section{RESULTS}

\section{Identification and Selection of Studies}

We identified 1587 potentially eligible records by searching PubMed, Embase, and Cochrane Library from their inception to October 2020. A total of 1251 unique records were retained after removing duplicate records. After initially checking the eligibility of the remaining records based on title and abstract, 1188 ineligible records were excluded. We obtained 63 full texts to further check their eligibility. After evaluating the full texts, 50 studies were excluded for several reasons such as ineligible design and insufficient data, and then 13 eligible studies were considered to be eligible for our inclusion criteria. We also checked the reference lists of meta-analyses focused on the same topic and then added an additional seven eligible studies. Therefore, we finally included 20 


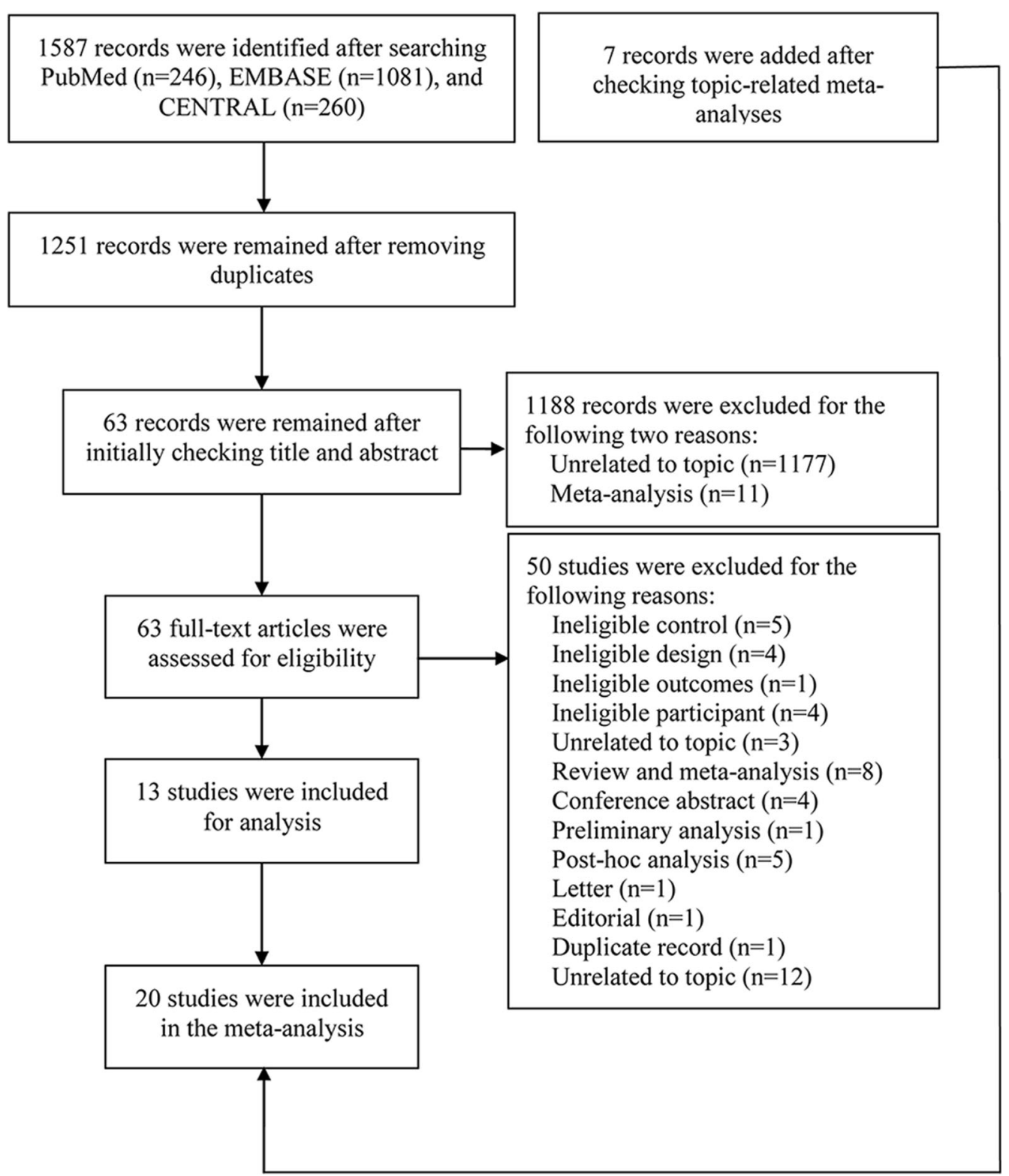

Fig. 1 Flow diagram of searching and selecting studies. We searched the Cochrane Library to identify potentially eligible studies indexed in CENTRAL. CENTRAL, Cochrane Central Register of Controlled Trials

eligible studies [11-13, 23-39] for the final analysis. The identification and selection of studies are shown in Fig. 1.

\section{Basic Characteristics of Eligible Studies}

These 20 eligible studies were published between 2001 and 2019. The total sample size of individual studies was between 50 and 1180, with a total sample size of 7804 . Of these 20 eligible studies, 12 [11, 27-37] investigated the comparative efficacy between linezolid and vancomycin, 5 [12, 23-26] focused on daptomycin vs. vancomycin, and 3 [13, 38, 39] compared tedizolid with linezolid. Although one study [11] had a three-arm design, treatment regimes in two arms fullfilled our inclusion criteria. Most of the eligible studies [11, 23-29, 32-38] (15 RCTs) had a multicenter 
design, and two studies [12, 30] definitively reported having a single-center design; the remaining 3 studies [13, 31, 39] did not report their design. Most studies (16 RCTs) were performed in the USA except for four studies that were performed in Japan [23, 28], Israel [34], and China [38], respectively. Details about these 20 eligible studies are summarized in Table 1.

\section{Risk of Bias}

Among the 20 included studies, 11 [11-13, 24, 26, 34-39] definitively reported the methods for generating random sequences such as computerized randomization, 6 studies [11-13, 25, 34, 39] performed appropriate allocation concealment, 8 studies [11, 13, 33-37, 39] correctly blinded participats, personnel, and outcome assessor, and only 1 study [29] had a high risk of bias related to incomplete outcome data. All studies were rated as having a low risk of bias in selective reporting and other bias. Overall, the level of risk of bias among all studies was considered to be low to moderate. The summary of the risk of bias is given in Figure S1.

\section{Clinical Success}

A total of 12 studies [11, 27-37], 5 studies [12, 23-26], and 3 studies [13, 38, 39] directly investigated the clinical success of linezolid vs. vancomycin, daptomycin vs. vancomycin, and tedizolid vs. linezolid, respectively. The evidence structure of these three comparisons is given in Fig. 2. Direct meta-analysis indicated a significant statistical difference between linezolid and vancomycin (OR, 1.46; 95\% CI, $1.07-1.99 ; I^{2}=49 \%, P=0.02$ ), and the remaining two comparisons including daptomycin vs. vancomycin and tedizolid vs. linezolid were not significantly different. The pooled results of the three comparisons in terms of clinical success are given in Fig. 3.

We also performed network meta-analysis to further investigate the comparative efficacy of these four antibiotics. The pooled result from the network meta-analysis based on the consistency model suggested that linezolid was superior to vancomycin in improving clinical success (OR, 1.59; 95\% CrI, 1.12 to 2.52 ), which was consistent with the finding of direct metaanalysis. The comparative efficacy of other remaining comparisons was not significantly different, which was also consistent with the findings of direct meta-analysis. All results of network meta-analysis of clinical success are summarized in Fig. 4A.

We generated ranking probabilities of all antibiotics in terms of clinical success. Results indicated that tedizolid had the highest probability of being ranked first, followed by linezolid, vancomycin, and daptomycin. The plot of rankings of all antibiotics is delineated in Fig. 5A.

\section{Microbiological Success}

A total of 11 studies [11, 27-33, 35-37], 3 studies [23, 25, 26], and 2 studies [38, 39] directly investigated the microbiological success of linezolid vs. vancomycin, daptomycin vs. vancomycin, and tedizolid vs. linezolid, respectively. Direct meta-analysis suggested that linezolid was superior to vancomycin in improving microbiological success (OR, 1.89; 95\% CI, 1.24-2.86; $I^{2}=68 \%, P=0.003$ ); however, no statistical difference was detected when daptomycin was compared to vancomycin or tedizolid related to linezolid. The pooled results of the three comparisons of linezolid vs. vancomycin, daptomycin vs. vancomycin, and tedizolid vs. linezolid in terms of microbiological success are delineated in Fig. 6.

We performed network meta-analysis to further confirm the findings from direct metaanalysis. Network meta-analysis based on the consistency model also indicated an improvement in microbiological success when linezolid was compared with vancomycin (OR, 1.95; 95\% CrI, 1.14-3.34), which was consistent with the finding of direct meta-analysis. Meanwhile, no statistical difference was detected regarding the remaining comparisons, which were also consistent with the findings of direct meta-analysis. All results of network meta-analysis of microbiological success are summarized in Fig. 4B. 


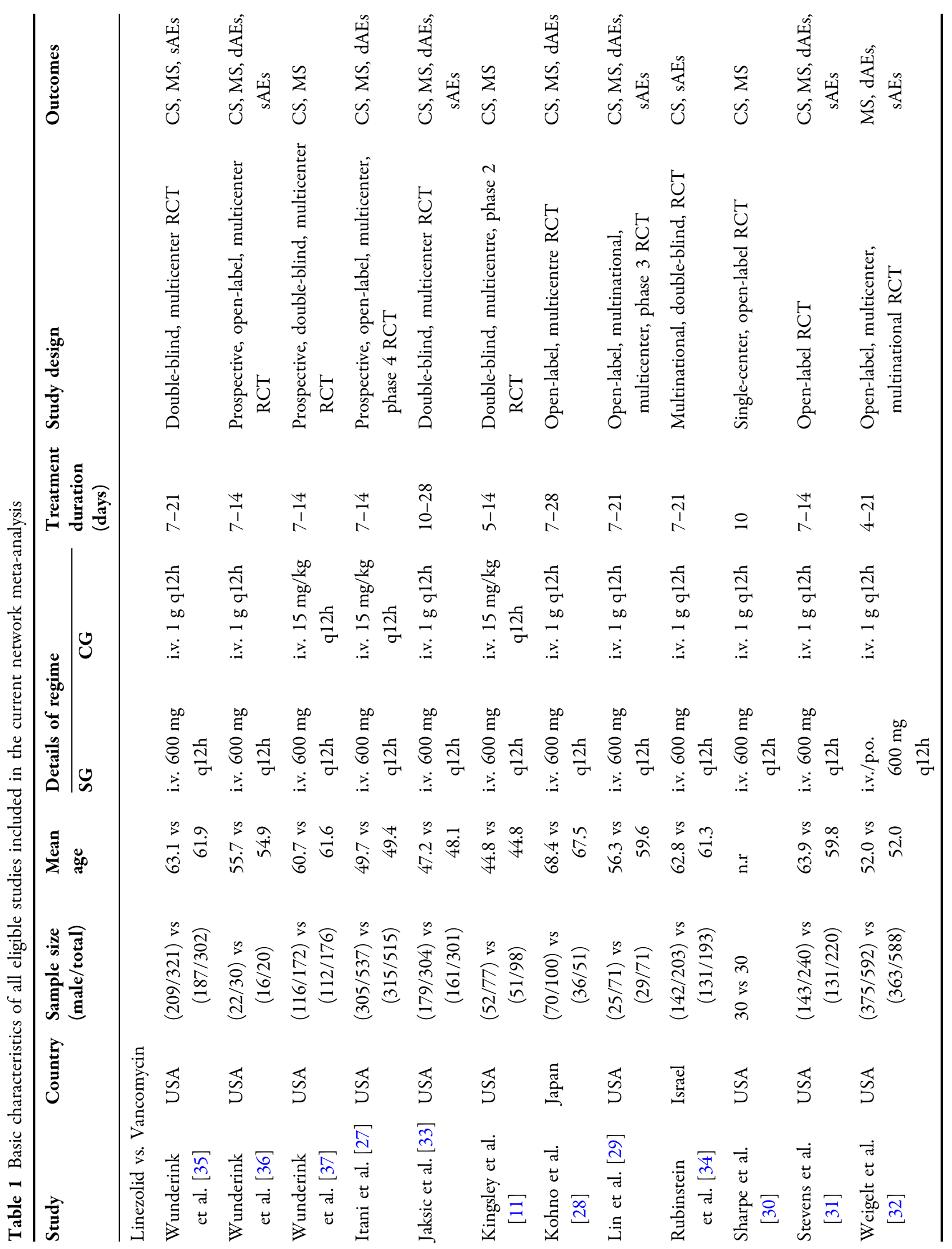




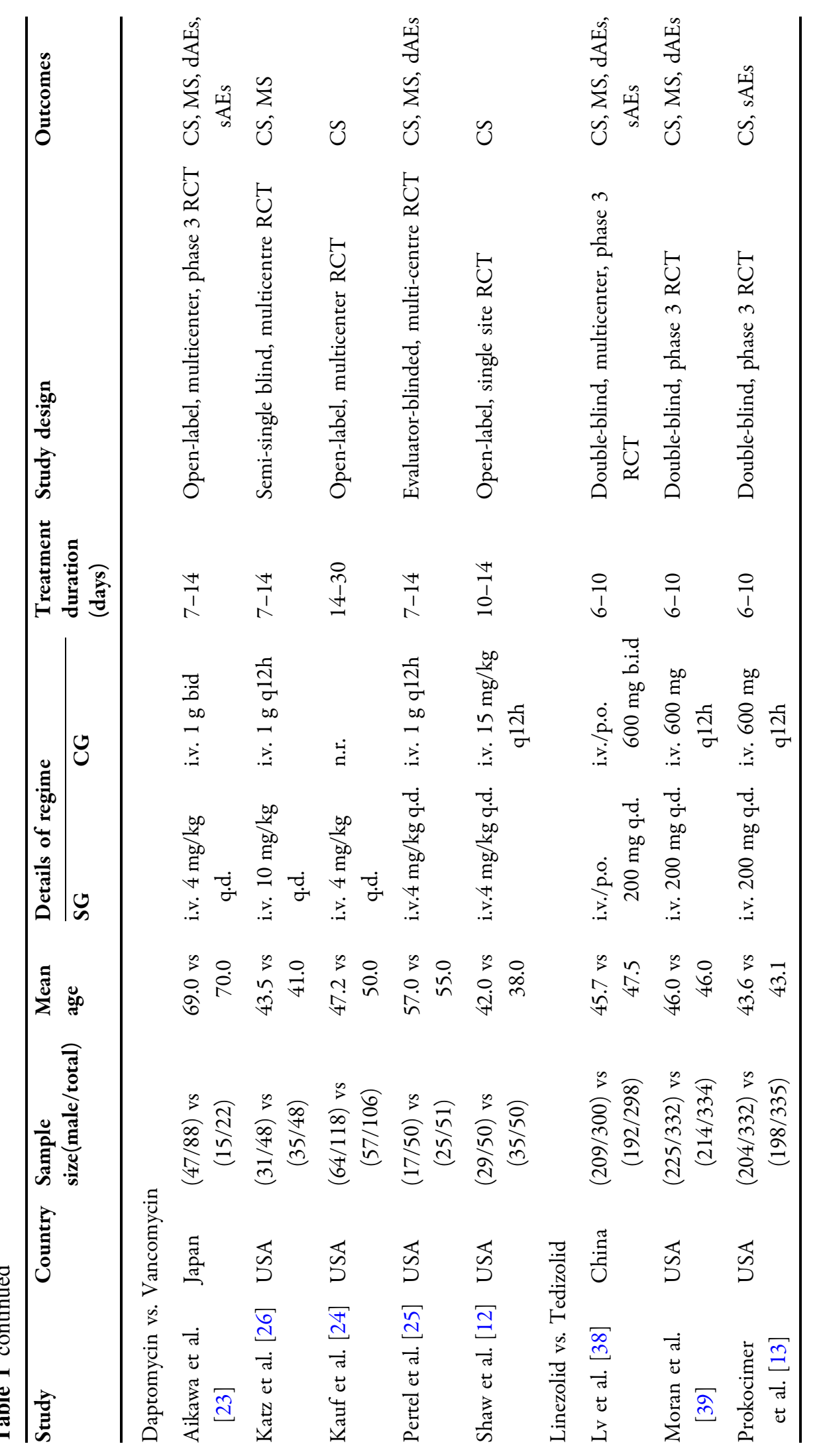


We generated ranking probabilities of all antibiotics in terms of microbiological success. Results indicated that linezolid had the highest probability of being ranked first, followed by tedizolid, vancomycin, and daptomycin. The plot of rankings of all antibiotics is delineated in Fig. 5B.

\section{Drug-Related Adverse Events}

A total of seven studies [27-29, 31-33, 36], seven studies [23, 25], and two studies [38, 39] directly investigated the drug-related AEs of linezolid vs. vancomycin, daptomycin vs. vancomycin, and tedizolid vs. linezolid, respectively. Direct meta-analysis did not suggest statistical difference among these three comparisons including linezolid vs. vancomycin, daptomycin vs. vancomycin, and tedizolid vs. linezolid in terms of drug-related AEs. The pooled results are given in Fig. 7.

Network meta-analysis was also performed to further confirm the findings from direct metaanalysis in terms of this outcome and obtained results consistent with direct meta-analysis. All results of network meta-analysis of drug-related AEs are summarized in Fig. 4C.

We generated ranking probabilities of all antibiotics in terms of drug-related AEs. Results indicated that daptomycin had the highest probability of being ranked first, followed by linezolid, vancomycin, and tedizolid for increasing the risk of drug-related adverse events. The plot of rankings of all antibiotics is delineated in Fig. 5C.

\section{Serious Adverse Events}

A total of seven studies $[29,31-36]$, one study [23], and two studies [13, 38] directly investigated the serious AEs of linezolid vs. vancomycin, daptomycin vs. vancomycin, and tedizolid vs. linezolid, respectively. Direct metaanalysis did not detect a statistical difference among these three comparisons including linezolid vs. vancomycin, daptomycin vs. vancomycin, and tedizolid vs. linezolid, which are delineated in Fig. 8.

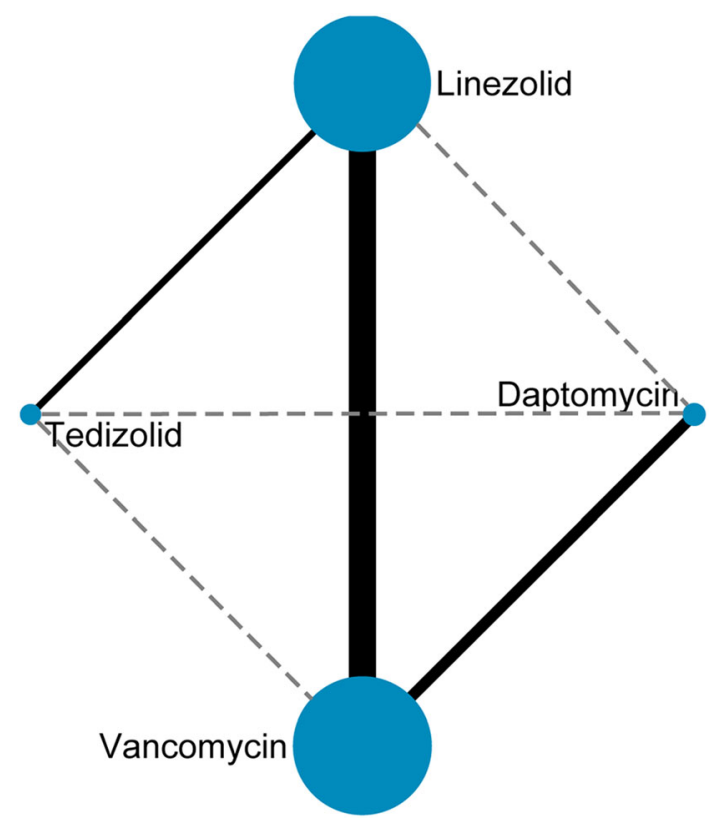

Fig. 2 Evidence structure of clinical success. The size of the node corresponds to the accumulated sample size of the individual antibiotic, and the thickness is positively associated with the accumulated number of eligible studies for individual comparison

Network meta-analysis based on the consistency model was also performed to further investigate the comparative efficacy of these four antibiotics and also suggested no statistical difference among all direct and indirect comparisons on serious AEs (Fig. 4D).

Ranking probabilities of all antibiotics were calculated to determine the ranking of individual antibiotics in terms of serious AEs. Results indicated that vancomycin had the highest probability of being ranked best, followed by linezolid, tedizolid, and daptomycin, for increasing the risk of serious AEs. The plot of rankings of all antibiotics is delineated in Fig. 5D.

\section{Publication Bias and Network Inconsistency}

We did not adopt the split-node method to check the network inconsistency because no first loop was constructed in the current network meta-analysis. However, the accumulated number of eligible studies for clinical success 


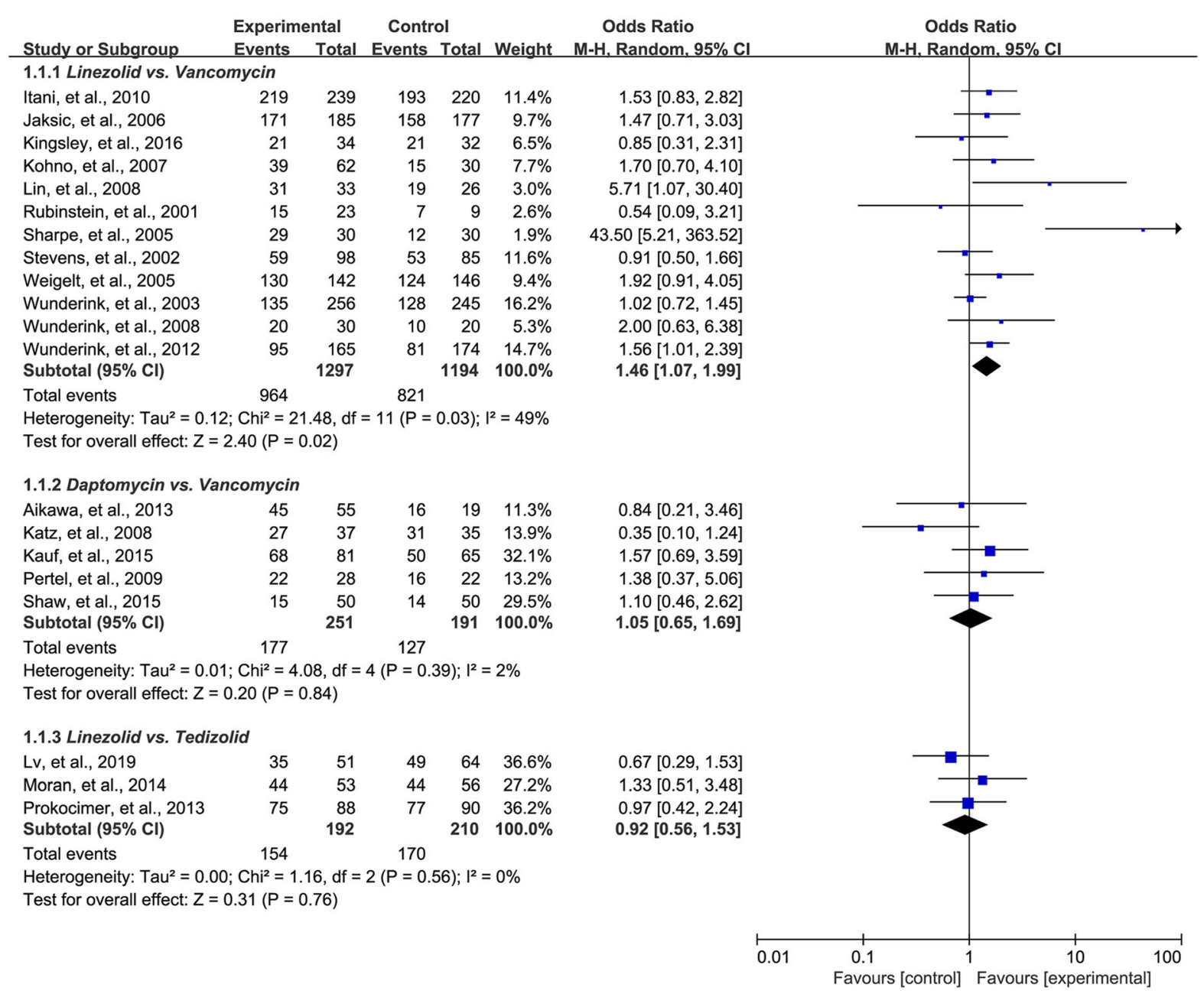

Fig. 3 Forest plot of clinical success

and microbiological success was $>10$; thus, we drew the funnel plot to qualitatively inspect whether publication bias was present or not. The funnel plot did not provide evidence of publication bias (Figure S2 and S3).

\section{DISCUSSION}

Methicillin-resistant Staphylococcus aureus (MRSA)-complicated SSTIs have been a threatening challenge worldwide, and oral or intravenous use of antibiotics remains the gold standard for this condition [8]. Although several clinical studies and meta-analyses have been performed to investigate the comparative efficacy and safety of different antibiotics for the treatment of MRSA infection, no definitive conclusion has been obtained. The current network meta-analysis aimed to determine the comparative efficacy and safety of vancomycin, linezolid, tedizolid, and daptomycin. The direct meta-analysis indicated that linezolid is associated with improved clinical and microbiological success compared to vancomycin, which is further established in network meta-analysis. For the other comparisons, no statistical difference was detected regarding all outcomes. 


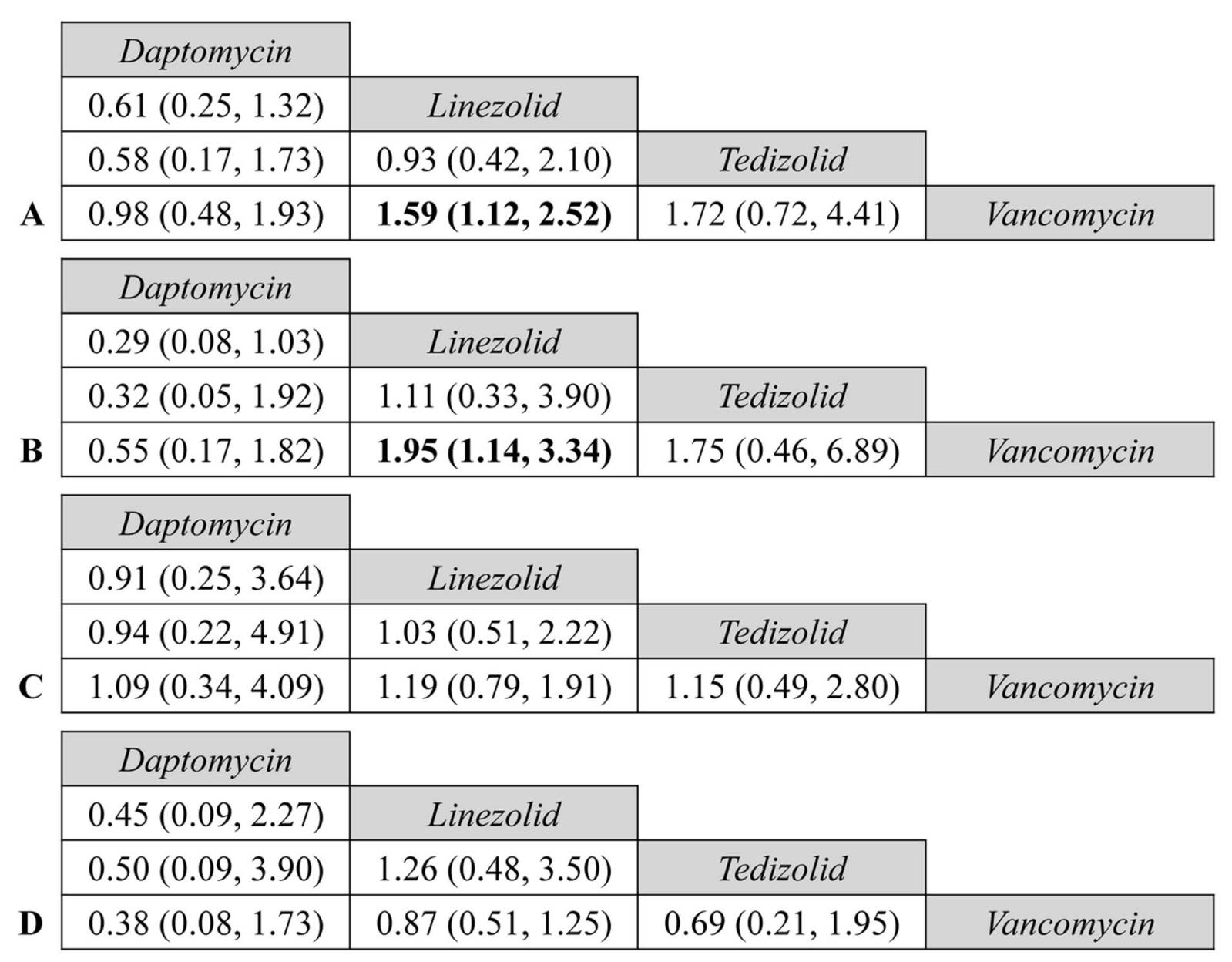

Fig. 4 Network meta-analysis of outcomes. The bold number indicates significant differences. A Clinical success. B Microbiological success. C Drug-related AEs. D Serious AEs. AEs, adverse events

To date, the four most recent meta-analyses $[15,16,40,41]$ investigated the role of the four antibiotics we focused on in the current network meta-analysis. In 2018, Li et al. [41] published a traditional direct meta-analysis of 11 RCTs to investigate the efficacy and safety of linezolid compared with other treatments for SSTIs and found linezolid was significantly superior to vancomycin in treating SSTIs, consistent with our finding. In fact, however, only eight eligible studies investigating comparative efficacy and safety between linezolid and vancomycin among adult patients were included after excluding a study focused on children [42]. In 2016, Liu et al. [40] performed a trial sequential meta-analysis to investigate the comparative efficacy and safety of daptomycin versus other antibiotics for SSTIs and found an equal potential of treating SSTIs between daptomycin and control antibiotics, which was also consistent with our finding. Although eight eligible studies were pooled in Liu's meta-analysis, the comparators among three studies were not vancomycin [43] or not only vancomycin $[44,45]$, which may limit the understanding of practitioners and decision makers of the true role of daptomycin in MRSA SSTIs compared to vancomycin. In 2019, Lan et al. [15] compared the efficacy and safety between tedizolid and linezolid for acute bacterial skin and skin 

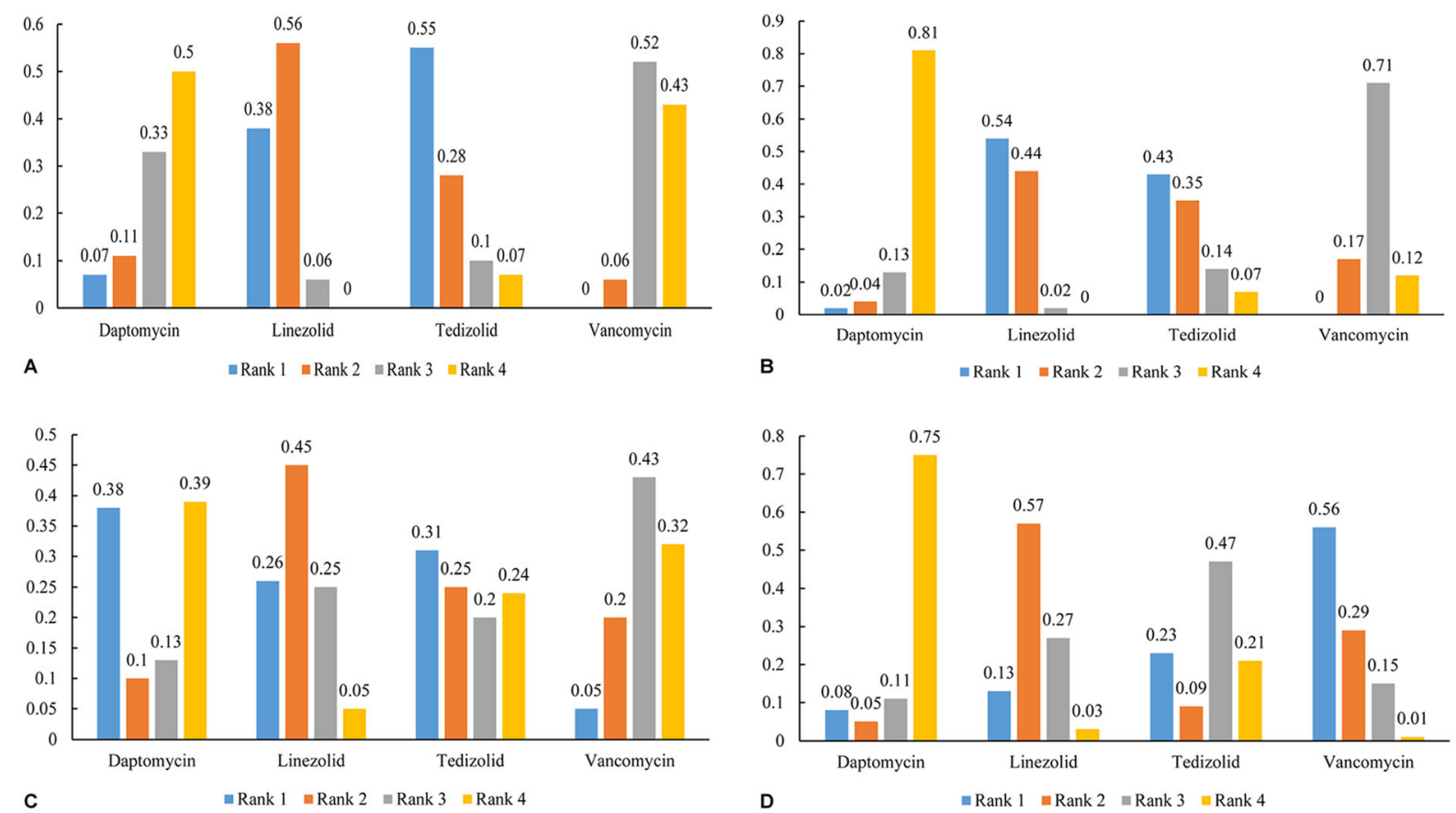

Fig. 5 Rank probability of all treatments for outcomes. A Clinical success; $\mathbf{B}$ microbiological success; C drugrelated AEs; D serious AEs. For positive outcomes including clinical success and microbiological success, ranking first indicates the highest probability of improving

structure infection (ABSSSI) and found a comparable efficacy and safety between these two antibiotics, which was further confirmed in our network meta-analysis. However, in this metaanalysis performed by Lan et al. [46], one study did not separately report infection caused by MRSA. In the same year, Zhang et al. [16] used the network meta-analysis method to investigate the comparative efficacy and safety of 16 antibiotics for the treatment of cSSTIs and hospital-acquired or ventilator-associated pneumonia. In this study, the authors found that linezolid was associated with clinical cure compared to vancomycin; however, no statistical difference between tedizolid and vancomycin was found. Unfortunately, some potentially eligible studies $[11,12,30,38]$ did not show any comparison of these three direct comparisons. Compared to the previous four

meta-analyses, the current network meta-analysis included more eligible studies and specifically investigated the role of each one of all four targeted antibiotics among patients with suspected or proven MRSA SSTIs. As a result, more comprehensive and robust evidence was generated from our network meta-analysis for evidence-based decisions.

Although the current network meta-analysis had several strengths such as a more comprehensive literature search, some limitations also must be further interpreted. First, no constructed first loop was identified, and thus some pooled results in the network meta-analysis were only generated from indirect evidence, which may impair the robustness of our findings. Second, treatments duration among eligible studies were different from one to another; however, we did not perform subgroup analysis 


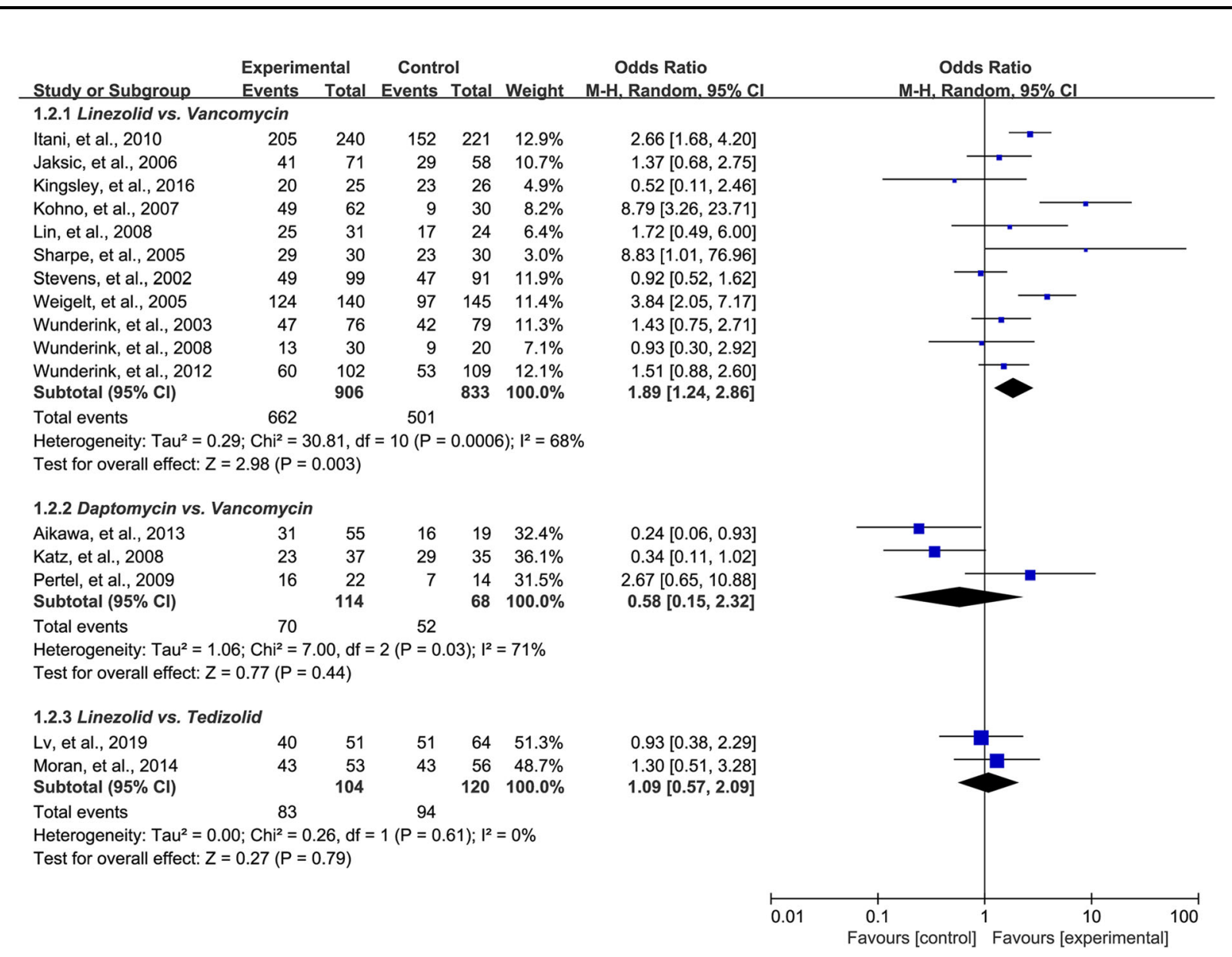

Fig. 6 Forest plot of microbiological success

to further investigate the impact of this factor on the pooled result. Third, study designs among the included studies were also different from one to another, which may also negatively affect the robustness of our pooled results because subgroup analysis was not performed. Certainly, we must interpret that limited data reduce the possibility of performing subgroup analysis in this network meta-analysis. Nevertheless, our study provided some promising findings for clinical decisions and further study designs although all results were generated from limited data. Finally, our network meta-analysis did not indicate whether patients receive an appropriate dose of vancomycin throughout the therapy to maintain the serum concentrations in the correct range or to prevent toxicity.

\section{CONCLUSIONS}

In summary, linezolid may be the preferred antibiotic for the treatment of MASR-related infection based on the limited evidence in order to improve the clinical and microbiological success and does not increase the incidence of drug-related and serious AEs. However, more high-quality studies must be performed to accurately determine the optimal treatment option. 


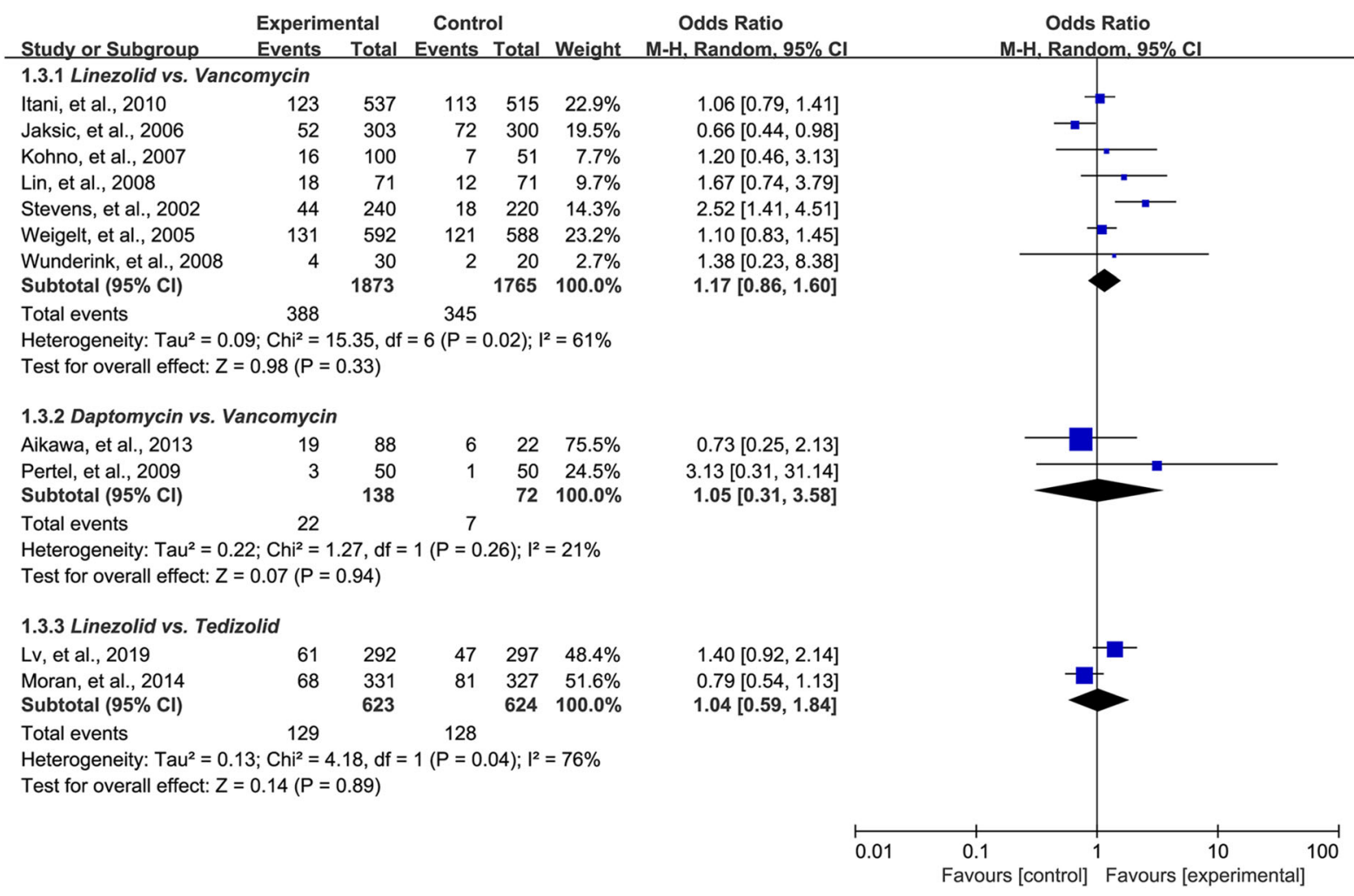

Fig. 7 Forest plot of drug-related AEs

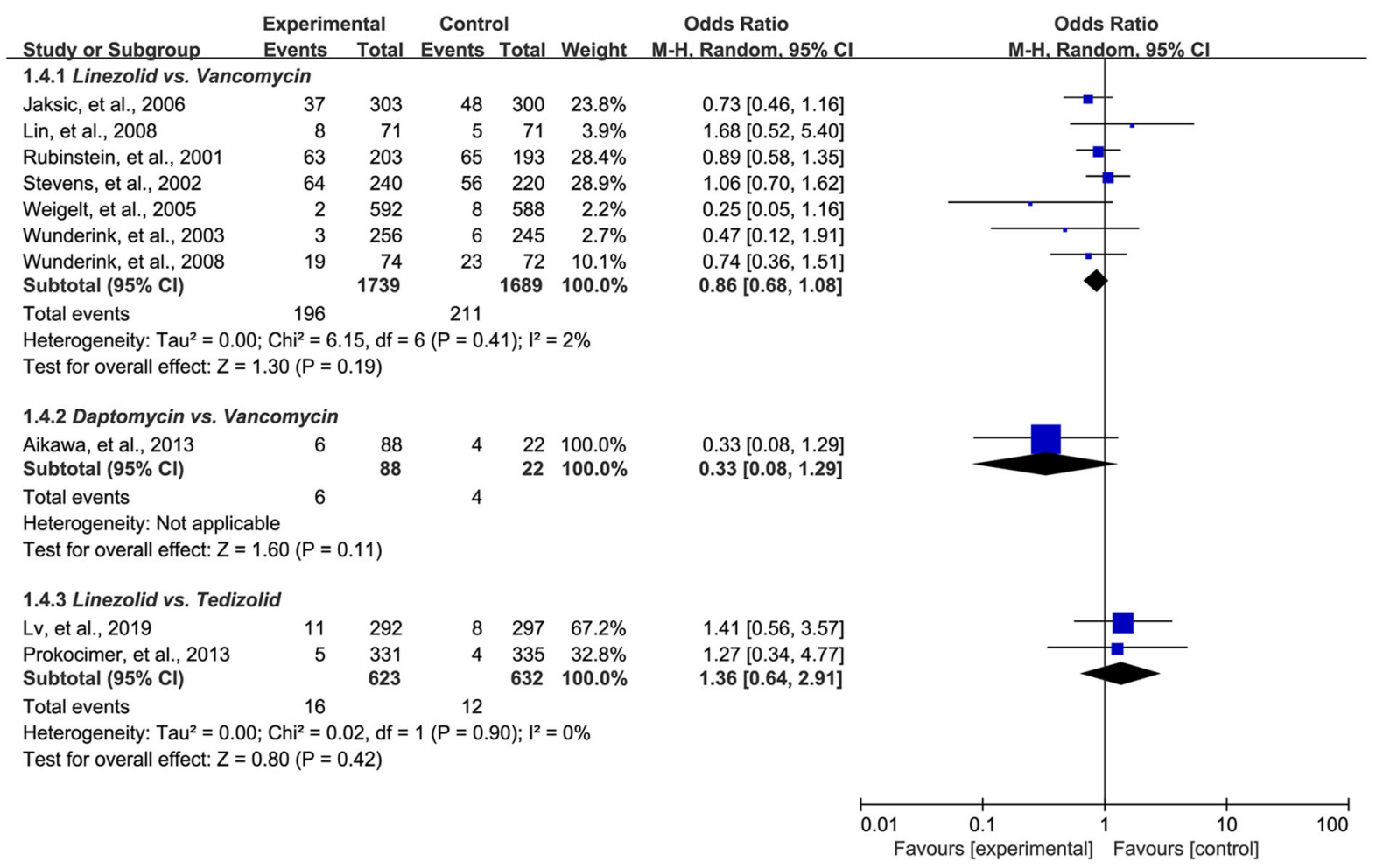

Fig. 8 Forest plot of serious AEs 


\section{ACKNOWLEDGEMENTS}

Funding. The Rapid Service Fee was funded by the authors.

Authorship. All named authors meet the International Committee of Medical Journal Editors (ICMJE) criteria for authorship for this article, take responsibility for the integrity of the work as a whole, and have given their approval for this version to be published.

Authors' Contributions. Substantially contributed to conception or design: Jingjuan Feng, Feng Xiang, Jian Cheng, Jun Li. Contributed to acquisition, analysis, or interpretation of data: Jingjuan Feng, Yeli Gou, Feng Xiang. Drafted the manuscript for important content: Jingjuan Feng, Feng Xiang, Jian Cheng, Yeli Gou. Critically revised the manuscript for important intellectual content: Feng Xiang, Jingjuan Feng., Jun Li. Gave final approval: All authors.

Disclosures. Jingjuan Feng, Feng Xiang, Jian Cheng, Yeli Gou, and Jun Li have nothing to disclose.

Compliance with Ethics Guidelines. This article is based on previously conducted studies and does not contain any new studies with human participants or animals performed by any of the authors.

Data Availability. All data generated or analyzed during this study are included in this published article/as supplementary information files.

Open Access. This article is licensed under a Creative Commons Attribution-NonCommercial 4.0 International License, which permits any non-commercial use, sharing, adaptation, distribution and reproduction in any medium or format, as long as you give appropriate credit to the original author(s) and the source, provide a link to the Creative Commons licence, and indicate if changes were made. The images or other third party material in this article are included in the article's Creative Commons licence, unless indicated otherwise in a credit line to the material. If material is not included in the article's Creative Commons licence and your intended use is not permitted by statutory regulation or exceeds the permitted use, you will need to obtain permission directly from the copyright holder. To view a copy of this licence, visit http://creativecommons.org/licenses/by$\mathrm{nc} / 4.0 /$.

\section{REFERENCES}

1. Bounthavong M, Hsu DI. Efficacy and safety of linezolid in methicillin-resistant Staphylococcus aureus (MRSA) complicated skin and soft tissue infection (cSSTI): a meta-analysis. Curr Med Res Opin. 2010;26:407-21.

2. National Nosocomial Infections Surveillance (NNIS) Report, data summary from October 1986-April 1996, issued May 1996: a report from the National Nosocomial Infections Surveillance (NNIS) System. Am J Infect Control. 1996;24:380-8.

3. Chambers HF. The changing epidemiology of Staphylococcus aureus? Emerg Infect Dis. 2001;7: 178-82.

4. Daum RS. Clinical practice. Skin and soft-tissue infections caused by methicillin-resistant Staphylococcus aureus. N Engl J Med. 2007;357:380-90.

5. Crum NF, Lee RU, Thornton SA, et al. Fifteen-year study of the changing epidemiology of methicillinresistant Staphylococcus aureus. Am J Med. 2006;119: 943-51.

6. Shorr AF. Epidemiology and economic impact of meticillin-resistant Staphylococcus aureus: review and analysis of the literature. Pharmacoeconomics. 2007;25:751-68.

7. McCollum M, Sorensen SV, Liu LZ. A comparison of costs and hospital length of stay associated with intravenous/oral linezolid or intravenous vancomycin treatment of complicated skin and softtissue infections caused by suspected or confirmed methicillin-resistant Staphylococcus aureus in elderly US patients. Clin Ther. 2007;29:469-77.

8. Tsoulas C, Nathwani D. Review of meta-analyses of vancomycin compared with new treatments for Gram-positive skin and soft-tissue infections: are we any clearer? Int J Antimicrob Agents. 2015;46: $1-7$. 
9. Cosgrove SE, Carroll KC, Perl TM. Staphylococcus aureus with reduced susceptibility to vancomycin. Clin Infect Dis. 2004;39:539-45.

10. Basseti M, Patel D, Chuang L, et al. An indirect comparison of clinical success of antimicrobial agents for confirmed methicillin-resistant Staphylococcus aureus (MRSA) complicated skin and soft-tissue infections (CSSTI). Surg Infect. 2013;14:A9.

11. Kingsley J, Mehra P, Lawrence LE, et al. A randomized, double-blind, Phase 2 study to evaluate subjective and objective outcomes in patients with acute bacterial skin and skin structure infections treated with delafloxacin, linezolid or vancomycin. J Antimicrob Chemother. 2016;71:821-9.

12. Shaw GJ, Meunier JM, Korfhagen J, et al. Randomized controlled noninferiority trial comparing daptomycin to vancomycin for the treatment of complicated skin and skin structure infections in an observation unit. J Emerg Med. 2015;49:928-36.

13. Prokocimer P, De Anda C, Fang E, Mehra P, Das A. Tedizolid phosphate vs linezolid for treatment of acute bacterial skin and skin structure infections: the ESTABLISH-1 randomized trial. JAMA. 2013;309:559-69.

14. Bally M, Dendukuri N, Sinclair A, Ahern SP, Poisson M, Brophy J. A network meta-analysis of antibiotics for treatment of hospitalised patients with suspected or proven meticillin-resistant Staphylococcus aureus infection. Int J Antimicrob Agents. 2012;40: 479-95.

15. Lan SH, Lin WT, Chang SP, et al. Tedizolid versus linezolid for the treatment of acute bacterial skin and skin structure infection: a systematic review and meta-analysis. Antibiotics (Basel). 2019;2019:8.

16. Zhang Y, Wang Y, Van Driel ML, et al. Network meta-analysis and pharmacoeconomic evaluation of antibiotics for the treatment of patients infected with complicated skin and soft structure infection and hospital-acquired or ventilator-associated penumonia. Antimicrob Resist Infect Control. 2019;8:72.

17. Salanti G. Indirect and mixed-treatment comparison, network, or multiple-treatments meta-analysis: many names, many benefits, many concerns for the next generation evidence synthesis tool. Res Synth Methods. 2012;3:80-97.

18. Higgins JPT, Green S. Cochrane handbook for systematic reviews of interventions version 5.1.0 [updated March 2011]. The Cochrane Collaboration, 2011. www.handbookcochraneorg. 2011.

19. Hutton B, Salanti G, Caldwell DM, et al. The PRISMA extension statement for reporting of systematic reviews incorporating network metaanalyses of health care interventions: checklist and explanations. Ann Intern Med. 2015;162:777-84.

20. Higgins JP, Altman DG, Gotzsche PC, et al. The Cochrane Collaboration's tool for assessing risk of bias in randomised trials. BMJ. 2011;343:d5928.

21. Cipriani A, Higgins JPT, Geddes JR, Salanti G. Conceptual and technical challenges in network meta-analysis. Ann Internal Med. 2013;159:130-7.

22. Page MJ, McKenzie JE, Higgins JPT. Tools for assessing risk of reporting biases in studies and syntheses of studies: a systematic review. BMJ Open. 2018;8:e019703.

23. Aikawa N, Kusachi S, Mikamo H, et al. Efficacy and safety of intravenous daptomycin in Japanese patients with skin and soft tissue infections. J Infect Chemother. 2013;19:447-55.

24. Kauf TL, McKinnon P, Corey GR, et al. An openlabel, pragmatic, randomized controlled clinical trial to evaluate the comparative effectiveness of daptomycin versus vancomycin for the treatment of complicated skin and skin structure infection. BMC Infect Dis. 2015;15:503.

25. Pertel PE, Eisenstein BI, Link AS, et al. The efficacy and safety of daptomycin vs. vancomycin for the treatment of cellulitis and erysipelas. Int J Clin Pract. 2009;63:368-75.

26. Katz DE, Lindfield KC, Steenbergen JN, et al. A pilot study of high-dose short duration daptomycin for the treatment of patients with complicated skin and skin structure infections caused by gram-positive bacteria. Int J Clin Pract. 2008;62:1455-64.

27. Itani KMF, Dryden MS, Bhattacharyya H, Kunkel MJ, Baruch AM, Weigelt JA. Efficacy and safety of linezolid versus vancomycin for the treatment of complicated skin and soft-tissue infections proven to be caused by methicillin-resistant Staphylococcus aureus. Am J Surg. 2010;199:804-16.

28. Kohno S, Yamaguchi K, Aikawa N, et al. Linezolid versus vancomycin for the treatment of infections caused by methicillin-resistant Staphylococcus aureus in Japan. J Antimicrob Chemother. 2007;60: 1361-9.

29. Lin DF, Zhang YY, Wu JF, et al. Linezolid for the treatment of infections caused by gram-positive pathogens in China. Int $\mathrm{J}$ Antimicrob Agents. 2008;32:241-9.

30. Sharpe JN, Shively EH, Polk HC Jr. Clinical and economic outcomes of oral linezolid versus intravenous vancomycin in the treatment of MRSAcomplicated, lower-extremity skin and soft-tissue 
infections caused by methicillin-resistant Staphylococcus aureus. Am J Surg. 2005;189:425-8.

31. Stevens DL, Herr D, Lampiris H, Hunt JL, Batts DH, Hafkin B. Linezolid versus vancomycin for the treatment of methicillin-resistant Staphylococcus aureus infections. Clin Infect Dis. 2002;34:1481-90.

32. Weigelt J, Itani K, Stevens D, Lau W, Dryden M, Knirsch C. Linezolid versus vancomycin in treatment of complicated skin and soft tissue infections. Antimicrob Agents Chemother. 2005;49:2260-6.

33. Jaksic B, Martinelli G, Perez-Oteyza J, Hartman CS, Leonard LB, Tack KJ. Efficacy and safety of linezolid compared with vancomycin in a randomized, double-blind study of febrile neutropenic patients with cancer. Clin Infect Dis. 2006;42:597-607.

34. Rubinstein E, Cammarata S, Oliphant T, Wunderink R. Linezolid (PNU-100766) versus vancomycin in the treatment of hospitalized patients with nosocomial pneumonia: a randomized, double-blind, multicenter study. Clin Infect Dis. 2001;32:402-12.

35. Wunderink RG, Cammarata SK, Oliphant TH, Kollef $\mathrm{MH}$. Continuation of a randomized, doubleblind, multicenter study of linezolid versus vancomycin in the treatment of patients with nosocomial pneumonia. Clin Ther. 2003;25:980-92.

36. Wunderink RG, Mendelson MH, Somero MS, et al. Early microbiological response to linezolid vs vancomycin in ventilator-associated pneumonia due to methicillin-resistant Staphylococcus aureus. Chest. 2008;134:1200-7.

37. Wunderink RG, Niederman MS, Kollef MH, et al. Linezolid in methicillin-resistant Staphylococcus aureus nosocomial pneumonia: a randomized, controlled study. Clin Infect Dis. 2012;54:621-9.

38. Lv X, Alder J, Li L, et al. Efficacy and safety of tedizolid phosphate versus linezolid in a randomized phase 3 trial in patients with acute bacterial skin and skin structure infection. Antimicrob Agents Chemother. 2019;2019:63.
39. Moran GJ, Fang E, Corey GR, Das AF, De Anda C, Prokocimer P. Tedizolid for 6 days versus linezolid for 10 days for acute bacterial skin and skin-structure infections (ESTABLISH-2): a randomised, double-blind, phase 3, non-inferiority trial. Lancet Infect Dis. 2014;14:696-705.

40. Liu C, Mao Z, Yang M, et al. Efficacy and safety of daptomycin for skin and soft tissue infections: a systematic review with trial sequential analysis. Ther Clin Risk Manag. 2016;12:1455-66.

41. Li Y, Xu W. Efficacy and safety of linezolid compared with other treatments for skin and soft tissue infections: a meta-analysis. Biosci Rep. 2018;2018: 38.

42. Yogev R, Patterson LE, Kaplan SL, et al. Linezolid for the treatment of complicated skin and skin structure infections in children. Pediatr Infect Dis J. 2003;22:\$172-7.

43. Evers R, Antony NL, Ogechika Alozie MD, Suresh Antony MD. Pilot study comparing daptomycin and telavancin in the treatment of skin and soft tissue infections. Internet J Infect Dis. 2013;4: 3725-7.

44. Quist SR, Fierlbeck G, Seaton RA, Loeffler J, Chaves RL. Comparative randomised clinical trial against glycopeptides supports the use of daptomycin as first-line treatment of complicated skin and softtissue infections. Int J Antimicrob Agents. 2012;39: 90-1.

45. Arbeit RD, Maki D, Tally FP, Campanaro E, Eisenstein BI. The safety and efficacy of daptomycin for the treatment of complicated skin and skin-structure infections. Clin Infect Dis. 2004;38:1673-81.

46. Mikamo H, Takesue Y, Iwamoto Y, et al. Efficacy, safety and pharmacokinetics of tedizolid versus linezolid in patients with skin and soft tissue infections in Japan-results of a randomised, multicentre phase 3 study. J Infect Chemother. 2018;24:434-42. 